\title{
La structure tarifaire SwissDRG évolue-t-elle assez vite dans la mouvance du nouveau financement hospitalier?
}

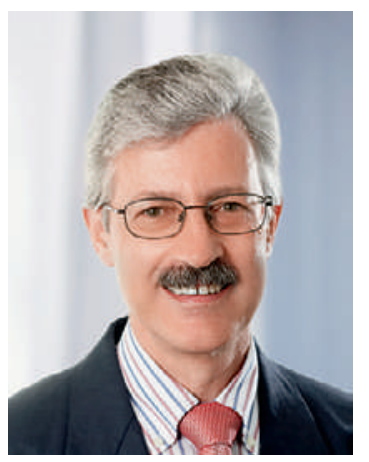

Depuis un peu plus d'un an, le nouveau financement hospitalier a profondément bousculé les anciennes habitudes. La très forte emprise étatique a diminué au profit d'une concurrence plus ouverte, conformément aux vœux du législateur. Cette bascule comporte naturellement des risques de surexploitation de créneaux rentables, au détriment des procédures complexes et exigeantes en termes de force de travail et de disponibilité. Les cantons, dont la charge a ainsi augmenté de plus de 1 milliard de francs par an, perdent de leur influence et en ressentent une légitime frustration. Eux-mêmes aux prises avec des budgets sanitaires serrés, ils y consacrent l'essentiel de leurs ressources à la participation aux factures hospitalières, n'ayant alors plus que de modestes montants disponibles pour l'indemnisation des prestations d'intérêt général. Bon nombre d'entre elles, telles que les services d'urgence, la médecine pénitentiaire ou certaines mesures psychiatriques, sont subitement menacées. Dans ce climat d'incertitude et bien que leur charge s'allège de manière significative, les assurances complémentaires ne baissent pas leurs primes, donnant ainsi de l'eau au moulin des détracteurs de notre système de santé, qui ne voient en lui qu'un centre de profit au détriment des patients.

\section{Il est primordial que la structure tarifaire soit représentative des coûts réels nécessaires à une prise en charge correcte des malades.}

Face à cette évolution, il est donc primordial que la structure tarifaire SwissDRG soit la plus représentative possible des coûts réels nécessaires à une prise en charge correcte des malades. La version 2.0 présentement en vigueur ne remplit pas encore suffisamment cette exigence. Conscient de cette réalité, le Conseil d'administration de SwissDRG SA a défini sa stratégie pour les années à venir: le besoin d'affiner le système a été reconnu. En effet, les hôpitaux publics à vocation centrale pratiquant une médecine très spécialisée, particulièrement les établissements universitaires, sont actuellement pré- térités. Pour y remédier, l'inclusion d'un nombre significatif de rétributions additionnelles est indispensable. Cependant, pour des raisons de congruence et afin de coller au mieux à la réalité du pays, il a été décidé de n'implémenter que des positions calculées sur la base de données helvétiques. Ceci prend du temps et dépend fortement de la qualité des données fournies par nos hôpitaux. La version 3.0 verra ainsi l'apparition d'une série de nouvelles rétributions additionnelles.

\section{Des cas à coûts très élevés sont suscep- tibles de grever considérablement l'équilibre financier d'un hôpital.}

La thématique des innovations a également été retravaillée, avec prise de conscience du fait que la procédure actuelle n'est pas satisfaisante. En effet, des négociations entre partenaires tarifaires sont toujours possibles avant l'implémentation d'une nouvelle thérapie dans la structure: ceci est cependant extrêmement fastidieux et souvent, le traitement d'un patient ne peut pas attendre le résultat de cette tractation avant d'être administré. Le risque d'absence de couverture de ces frais est alors très grand pour les prestataires de soins.

Enfin, la particularité des cas à coûts très élevés a été relevée. Bien que peu nombreux, ils sont cependant susceptibles de grever considérablement l'équilibre financier d'un hôpital, quelle que soit sa taille. Une solution a été proposée, pas encore entièrement aboutie (cf. l'article de P. Ingenpass à ce sujet, à la page 319 de ce numéro).

Les premiers bilans annuels 2012 vont bientôt être publiés. Conjointement avec les difficultés rencontrées partout dans les négociations du taux de base 2013, ils vont engendrer une nouvelle vague de rationalisation, à certains endroits nécessaire mais parfois douloureuse. Face à ceci et pour éviter que les hôpitaux efficients ne soient pénalisés, la FMH s'engage résolument, dans le cadre de SwissDRG SA, pour améliorer encore la crédibilité de notre structure tarifaire. C'est ici également l'occasion de remercier notre Casemix office qui, avec des moyens limités, effectue sereinement un énorme travail de grande qualité.

Dr Pierre-François Cuénoud, vice-président de la FMH, responsable du domaine Tarifs et économie de la santé pour les médecins hospitaliers 